\title{
Guided Search: An Alternative to the Feature Integration Model for Visual Search
}

\author{
Jeremy M. Wolfe, Kyle R. Cave, and Susan L. Franzel \\ Department of Brain and Cognitive Sciences \\ Massachusetts Institute of Technology
}

\begin{abstract}
Subjects searched sets of items for targets defined by conjunctions of color and form, color and orientation, or color and size. Set size was varied and reaction times (RT) were measured. For many unpracticed subjects, the slopes of the resulting RT $\times$ Set Size functions are too shallow to be consistent with Treisman's feature integration model, which proposes serial, self-terminating search for conjunctions. Searches for triple conjunctions (Color $\times$ Size $\times$ Form) are easier than searches for standard conjunctions and can be independent of set size. A guided search model similar to Hoffman's (1979) two-stage model can account for these data. In the model, parallel processes use information about simple features to guide attention in the search for conjunctions. Triple conjunctions are found more efficiently than standard conjunctions because three parallel processes can guide attention more effectively than two.
\end{abstract}

Searches for a target among a number of distractor items are easier for some stimuli than for others. For example, targets defined by a unique color or a unique orientation are found easily (Tresiman \& Gelade, 1980). If we measure the time required to determine that a target is present, we find that the reaction time (RT) is short and nearly independent of the number of distractor items. The target, if present, appears to be found in "parallel" with the visual system examining all items at once. Other searches are not so effortless. A $T$ can be found among a field of $L s$, but the time required to find that $T$ will increase markedly as the number of distracting $L s$ increases (Julesz \& Bergen, 1983). The slope of the function relating RT to number of distractors gives an estimate of the cost in search time of each additional distractor. In a $T$ versus $L$ task, additional items seem to be processed at a rate of about $40-60 \mathrm{~ms}$ apiece (e.g., Julesz \& Bergen, 1983).

If the $T$ is located by a "serial," self-terminating search (Donders, 1868/1969; Sternberg, 1969), a 2:1 ratio in slopes is predicted between RT $\times$ Set Size functions for trials with a target present and blank trials containing only distractors. On blank trials, the subject must examine each item in order to confirm that no target is present. This yields a slope of 40 $\mathrm{ms} / \mathrm{item}$. On target trials, the subject must examine an average of half of the items before finding the target, yielding a slope of $20 \mathrm{~ms} / \mathrm{item}$. (The terms serial and parallel must be used with caution. $)^{\prime}$

We thank Amy Shorter, Charles Pokorny, and Art Figel for help in data collection and analysis, and Anne Treisman, David Irwin, James Pomerantz, Karen Yu, Nancy Kanwisher, and Jeff Schall for useful comments on drafts of this article. This research was supported by National Institutes of Health Grant No. EY05087, the Whitaker Health Sciences Fund, and the Educational Foundation of America. We thank IBM for the use of YODA graphics hardware and software.

Correspondence concerning this article should be addressed to Jeremy M. Wolfe, Department of Brain and Cognitive Sciences, E10137, Massachusetts Institute of Technology, Cambridge, Massachusetts 02139 .
Treisman's feature integration model (Treisman \& Gelade, 1980; Treisman, 1986), perhaps the leading model of visual search, seeks to explain the distinction between serial and parallel searches with a two-stage model. A fairly limited, "preattentive" (Neisser, 1967), parallel stage of processing is followed by a more sophisticated, serial stage. Treisman holds that only basic features such as color, size, and orientation can support parallel search, whereas all other stimuli require a serial search. In particular, she has argued that serial search is required for targets defined by conjunctions of basic features (e.g., a red $X$ among green $X$ s and red $O s$ ). Treisman has presented an extensive set of experiments showing fairly steep, linear RT $\times$ Set Size functions with 2:1 ratios between slopes for target and blank trials (Treisman \& Gelade, 1980; Treisman \& Paterson, 1984). Julesz's texton model (Julesz, 1981; Julesz, 1984; Julesz \& Bergen, 1983) shares many important features with the feature integration model.

It is a curious feature of these models that the parallel processes seem to have very little influence on the subsequent serial processes. In the standard feature integration model, the parallel processes can identify targets on the basis of a single feature. However, if they do not find a target, none of

\footnotetext{
' The terms serial and parallel must be used with some care. Townsend $(1971,1976)$ has shown that results such as those described above do not by themselves prove that an underlying search process is serial or parallel. For example, it may be that all searches are parallel and that the differences between searches lie in capacity limits on different parallel processes: a very large capacity for processes involved in identifying color, and more limited capacity for processes involved in more complex identifications (e.g., $T$ vs. $L$ ). Whether the underlying distinction is between serial and parallel processes or between capacity limited and unlimited processes, there remains an interesting, qualitative difference between effortless and effortful searches. For the sake of convenience, we will use serial to refer to searches that produce RT $\times$ Set Size functions with a substantial positive slope, and parallel to refer to functions with little or no slope We recognize that these are labels and not absolute commitments to a particular view of the nature of the underlying mechanisms.
} 
the information that they have gathered is used by the serial processes, even if that information might be useful. Consider a search for a red $X$ among green $X$ s and red $O$ s. The target is defined by a conjunction; therefore, it cannot be located by the parallel processes. Nevertheless, a parallel process for color can differentiate between green and red items. Because no green item can possibly be a red $X$, it would seem sensible for the parallel process to inform the serial process of the locations of all green items so that the serial process would not waste time and effort examining those items. Indeed, there is ample evidence that information from parallel processing of color can be used to restrict serial searches to items of a single color in a multicolored array (Bundesen \& Pedersen, 1983; Egeth, Virzi, \& Garbart, 1984; Farmer \& Taylor, 1980; Green \& Anderson, 1956; Smith, 1962).

In this article we present data from a series of visual search experiments suggesting that serial visual search can be guided by information from any of a number of parallel processes. In the first series of experiments, unpracticed subjects searched for conjunctions of color and form or color and orientation. In general, the slopes of the RT $\times$ Set Size functions are quite shallow. For many subjects, the slopes are virtually flat, or at least as "flat" as published data for feature searches.

There have been several other published reports of shallow slopes for conjunction searches. For example, Nakayama and Silverman (1986) found that searches for a number of conjunctions involving motion, stereoscopic depth, or both can produce very shallow $R T \times$ Set Size slopes (see also Steinman, 1987, and McLeod, Driver, \& Crisp, 1988). It has been possible to regard each of these previous cases as an exception to the general feature integration rule that conjunctions require serial search. For example, Nakayama and Silverman's data suggest that depth may behave in special ways as a feature. However, the results presented in this article will show that very shallow slopes can be obtained using the same classes of conjunctions (e.g., Color $\times$ Form) used by Treisman (Treisman \& Gelade, 1980) in formulating the feature integration model.

This raises the question of why our results differ from previously published results for similar conjunction searches. The second set of experiments addresses that issue. The results of Experiment 7 will show that the difference is largely attributable to differences in the stimuli. It is not due to learning (Experiments 5 and 6) or to a general ability of our subjects to do all searches in parallel (Experiment 4). Regardless of the explanation of the differences between our data and previously published results, it is important to realize that the simple existence of our data requires a modification of the feature integration model. That model holds that conjunctions (at least of color and form) require serial search. Our data show that in some cases this is not so.

In our modification of the feature integration model, we propose that the parallel processes guide the "spotlight of attention" toward likely targets. Thus, we call it "guided search." This is not an entirely new idea. Hoffman (1978, 1979) proposed a two-stage model in which a parallel first stage delivers likely targets to a slower, serial, second stage. Although the basic architecture of our proposal is similar to that of Hoffman's, his model is based on work from a somewhat different search task and does not deal explicitly with searches for conjunctions. An advantage of the guided search model is its ability to explain many of the previously published problematical results as examples of, and not exceptions to, the general feature integration rule.

The guided search model makes testable predictions. One such prediction is that triple conjunctions (Quinlan \& Humphreys, 1987) should be easier to find than standard conjunctions. If the parallel processes can guide subsequent serial search, then three parallel sources of guidance ought to be better than two. The standard feature integration model would predict serial search for such stimuli. In the third set of experiments, subjects searched for a triple conjunction of color, form, and size. These searches produce shallower slopes than simple Color $\times$ Form conjunctions. Indeed, in one condition, search for triple conjunctions is independent of set size.

To summarize, this article makes three main points:

1 . In our experiments, results from naive, unpracticed subjects searching for conjunctions of Color $\times$ Form, Color $\times$ Orientation, and Color $\times$ Size are inconsistent with serial, self-terminating search.

2. Searches for triple conjunctions are easier than for simple conjunctions, a fact not predicted by the standard feature integration model.

3. A modification of that model to allow the parallel processes to guide serial search can explain these and other problematical results.

\section{Experiment 1: Conjunctions of Color and Form}

\section{Method}

Subjects. Twenty subjects were tested. They were drawn from the Massachusetts Institute of Technology (MIT) undergraduate subject pool and were paid for their participation. All wore their best optical correction, if they required any. All were naive as to the purposes and method of the experiment. None had been subjects in previous visual search experiments.

Apparatus and procedure. Stimuli were presented on a standard television monitor that was part of a modified "Sub-Roc 3-D" video game. Displays were controlled by an IBM PC-XT with IBM-YODA graphics. Stimuli were saturated red and green $X \mathrm{~s}$ and $O$ s on a black background. (CIE, International Commission on Color, $x, y$ coordinates: red, $.62, .36$; green, $.34, .57)$. Subjects viewed an $11.3^{\circ}$ by $11.3^{\circ}$ field with a small central fixation point. Individual items fit within an $0.85^{\circ}$ by $0.85^{\circ}$ square. They could be placed at any of 36 locations in a slightly irregular 6 by 6 array. On each trial, items were presented at 8,16 , or 32 randomly chosen loci within the array. On target trials, one of these loci contained a target item. Set size, positions of target and distractors, and presence or absence of a target were random across trials. Subjects responded by pressing one of two keys: A yes key if a target was detected and a no key if it was not. Reaction times were measured from stimulus onset. The stimulus remained visible until the subject responded and feedback was given on each trial. Targets were present on $50 \%$ of trials. All experiments in this article were variations of this visual search paradigm.

In Experiment 1, each subject was run in one session of 260 trials. For the first 40 trials, subjects did a very simple search in order to 
familiarize themselves with the apparatus (e.g., a search for a purple square among green $X \mathrm{~s}$ ). In the next 220 trials, the target was a red $O$ and the distractors were green $O s$ and red $X \mathrm{~s}$. Thus, the target was defined by a conjunction of color and form. These stimuli were similar to those in Treisman and Gelade's (1980) Experiment 1. The first 20 trials were discarded as practice and the data from the remaining 200 used to derive $R T \times$ Set Size functions.

\section{Results}

Linear regressions for RT as a function of set size were computed separately for each subject for target and blank trials. All subjects showed quite linear results $(r>0.9$, though $r$ values are high for almost any monotonic set of three points, see Experiment 2). Table 1 gives the slopes for target and blank trials for all subjects in descending rank order by the slope for target trials. The final column gives ratio of blank trial to target trial slopes. The slopes are shallow. Half of our subjects have slopes of less than $6.0 \mathrm{~ms} /$ item on positive trials. In contrast, Treisman and Gelade (1980) obtained average slopes of 28.7 for positive and 67.1 for negative trials. Several of our subjects have slopes of 2-4 ms/item. These shallow slopes are comparable to Treisman and Gelade's (1980) slopes for feature searches. Average $y$-intercepts for RT $\times$ Set Size functions are $451 \mathrm{~ms}$ for target trials and $531 \mathrm{~ms}$ for blank trials.

Though they are shallow, the slopes are clearly positive. For target trials, RT increases linearly with set size, $F(1,38)=$ $100.1, p<.001$, with a slope of $7.5 \mathrm{~ms} /$ item. For blank trials, the linear increase is also highly significant, $F(1,38)=63.8$, $p<.001$, and the slope is $12.6 \mathrm{~ms} / \mathrm{item}$. The slopes for target and blank trials differ significantly from each other, as is

Table 1

Conjunctions of Color and Form: Individual Slopes for Target and Blank Trials

\begin{tabular}{|c|c|c|c|}
\hline Subject & $\begin{array}{c}\text { Target trials } \\
\text { (ms/item) }\end{array}$ & $\begin{array}{c}\text { Blank trials } \\
\text { (ms/item) }\end{array}$ & $\begin{array}{c}\text { Slope } \\
\text { ratio }\end{array}$ \\
\hline GN & 20.2 & 31.2 & 1.545 \\
\hline $\mathbf{J R}$ & 13.3 & 31.9 & 2.398 \\
\hline CS & 13.1 & 27.0 & 2.061 \\
\hline $\mathrm{CH}$ & 10.4 & 10.2 & 0.981 \\
\hline DDW & 9.2 & 30.3 & 3.293 \\
\hline SH & 8.1 & 6.4 & 0.79 \\
\hline DB & 8.0 & 14.0 & 1.75 \\
\hline JD & 7.9 & 17.0 & 2.152 \\
\hline MMM & 6.7 & 4.5 & 0.672 \\
\hline BW & 6.7 & 2.9 & 0.433 \\
\hline KG & 6.0 & 9.7 & 1.617 \\
\hline DG & 5.8 & 5.5 & 0.948 \\
\hline $\mathbf{R S}$ & 5.7 & 5.4 & 0.947 \\
\hline $\mathrm{JT}$ & 5.6 & 10.4 & 1.857 \\
\hline AD & 5.2 & 6.8 & 1.308 \\
\hline $\mathrm{AG}$ & 4.4 & 6.6 & 1.500 \\
\hline SS & 4.4 & 12.3 & 2.795 \\
\hline HD & 3.5 & 1.0 & 0.286 \\
\hline JMD & 3.3 & 6.9 & 2.091 \\
\hline $\mathbf{M M}$ & 2.0 & 12.7 & 6.350 \\
\hline$M$ & 7.5 & 12.6 & 1.8 \\
\hline$S D$ & 4.2 & 9.8 & 1.3 \\
\hline
\end{tabular}

shown by combining both sets of trials into a single analysis of variance (ANOVA) and testing the interaction between target presence and the linear increase with set size, $F(1,38)=17.4$, $p<.001$.

Are the slopes in the $2: 1$ ratio predicted by a serial selfterminating search? The mean slope ratio is 1.8 , which is not significantly lower than $2: 1, t(19)=0.69, p<.6$. Subject $M M$, however, has an extremely high slope ratio (6.4), which is almost twice the next highest value and more than three standard deviations above the mean. When this outlier is excluded, the mean slope ratio drops to 1.55 , and the difference from $2: 1$ is significant, $t(18)=2.43, p<.05$. If the ratio was $2: 1$, then two times the target trial slope should not be significantly different from the blank trial slope. For the 19 subjects (excluding MM), this hypothesis is rejected (paired $t$ test, $t=2.1, d f=18, p<.05$ ). Thus it seems likely that our subjects are not performing a standard serial self-terminating search.

The average error rates are $1.0 \%$ for set size $8,1.7 \%$ for 16 , and $3.8 \%$ for 32 . The error rate does rise significantly with set size, $F(2,57)=10.3, p<.001$. To examine the hypothesis that subjects having shallow slopes would make more errors, we performed correlations of error rate with slope. These are not significant for target trials $\left(r^{2}=0.17, p>0.05\right)$ or blank trials $\left(r^{2}=.16, p>.05\right)$. It might be proposed that subjects with shallow slopes made more errors only at the largest set sizes. However, the correlations of error rate at the largest set size with slope are not significant (target trials: $r^{2}=.18, p>$ .05 ; blank trials: $r^{2}=.13, p>.05$ ).

\section{Discussion}

Five conclusions might be drawn from these data: (1) Our results for conjunctions of color and form differ from those of Treisman and Gelade (1980). Our slopes are much shallower. We examined possible reasons for this difference in subsequent experiments. Regardless of the reason for the difference, the ability of our subjects to perform such efficient searches for conjunctions raises a problem for the feature integration model. (2) Given that shallow slopes are obtained with naive subjects and minimal (20 trials) practice, learning is not a likely explanation for our results (see Schneider \& Shiffrin, 1977; Shiffrin \& Schneider, 1977). Experiments 4 and 5 expand on this point. (3) Though we cannot reject the hypothesis that the slopes for blank and target trials are in a $2: 1$ ratio, that relationship does not appear to hold for the subjects with shallow slopes. (4) It would be possible to produce arbitrarily shallow slopes in any search task if the subjects simply waited some fixed amount of time before responding. This uninteresting outcome would result in long RTs (high $y$-intercepts). In Experiment 1, the $y$-intercepts are quite low (positive trials $=451 \mathrm{~ms}$, negative trials $=531 \mathrm{~ms}$ ), which is evidence against this explanation. (5) It would be possible to produce shallow slopes if subjects responded quickly but inaccurately (i.e., there would be a speed-accuracy trade-off). However, error rates are very low in this experiment. As we will show, the error rates are actually higher for tasks that produce slower, more serial patterns of RTs. 


\section{Experiment 2: Conjunctions of Color and Orientation}

The results from one experiment present an insufficient case for calling for a modification of a successful model. To bolster the case for a change in the feature integration model, we repeated the experiment using a slightly different conjunction (color and orientation) and different ranges of set sizes.

\section{Method}

In this case, subjects searched for a green, horizontal line among red horizontals and green verticals. A total of 22 subjects were tested. Some of these subjects had been in previous visual search experiments. Two of the authors served as subjects; the other 20 subjects were drawn from the MIT undergraduate subject pool. Subjects were divided into three groups, each of which was tested on a different group of set sizes: Group A, 3, 6, 9, 18, and 36; Group B, 1, 2, 4, 8, 16 , and 32; Group $C, 1,2,6,12$, and 24 . We used more than three set sizes per subject in order to better examine the linearity of the Set Size $\times$ RT function. Subjects received 20 practice trials and 100 experimental trials per set size. (Groups $A$ and $C$ received 520 trials and Group B received 620 .) In all other respects the methods were identical to those of the previous experiment.

\section{Results}

Figures $1 \mathrm{~A}$ and $1 \mathrm{~B}$ show average RTs for target and blank trials for each group of subjects. In general, the results replicate those from Experiment 1 . In all three versions of the experiment, RT shows a strong linear trend upward as set size increased, both for target trials [Group A, $F(1,35)=65.3$; Group B, $F(1,35)=182.6$; Group C, $F(1,20)=62.3 ; p<$ .001 in all cases] and for blank trials [Group $A, F(1,35)=$ 19.7; Group B, $F(1,35)=99.9$; Group C, $F(1,20)=54.5$; $p<.001$ in all cases]. Also for all three versions, negative slopes are steeper than positive slopes [Group A, $F(1,35)=$ 11.5; Group B, $F(1,35)=40.2$; Group C, $F(1,20)=10.3$; $p<.005$ in all cases].

As in Experiment 1, the RT $\times$ Set Size functions are substantially shallower than would be predicted if subjects were undertaking a simple, serial self-terminating search in which attention moved every $40 \mathrm{~ms}$. Average slopes for the three groups are given in Table 2.

Shallow as they are, the slopes for target trials are misleadingly high because, in this experiment, the target trial functions are not linear. In at least two of the three groups, the target slope decreases for larger set sizes, resulting in significant quadratic trends, Group B, $F(1,35)=20.1, p<.001$; Group $C, F(1,20)=6.6, p<.025$. The quadratic trend is not present in Group $\mathrm{A}(F<1)$, perhaps because the smaller set sizes were not included in that version or perhaps because the slopes for this set of subjects are so shallow that any nonlinearity is hidden. Given the nonlinear component in the target trial data, we did not attempt to test the hypothesis that the blank and target trial slopes were in a 2:1 ratio.

This negative acceleration of the RT function is apparent in Figure 1A. Table 3 shows slopes computed separately for the three lowest set sizes and the three highest set sizes. Clearly, slopes are steeper for smaller set sizes. For the higher set sizes, the slopes are very shallow, averaging 3-6 ms/item.

\section{A: Target Trials}

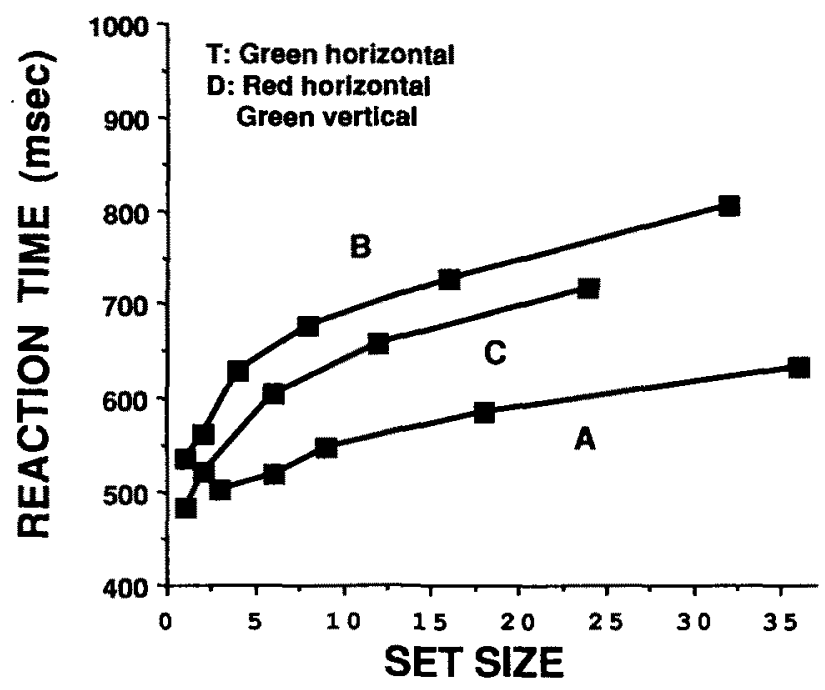

1B: Blank Trials

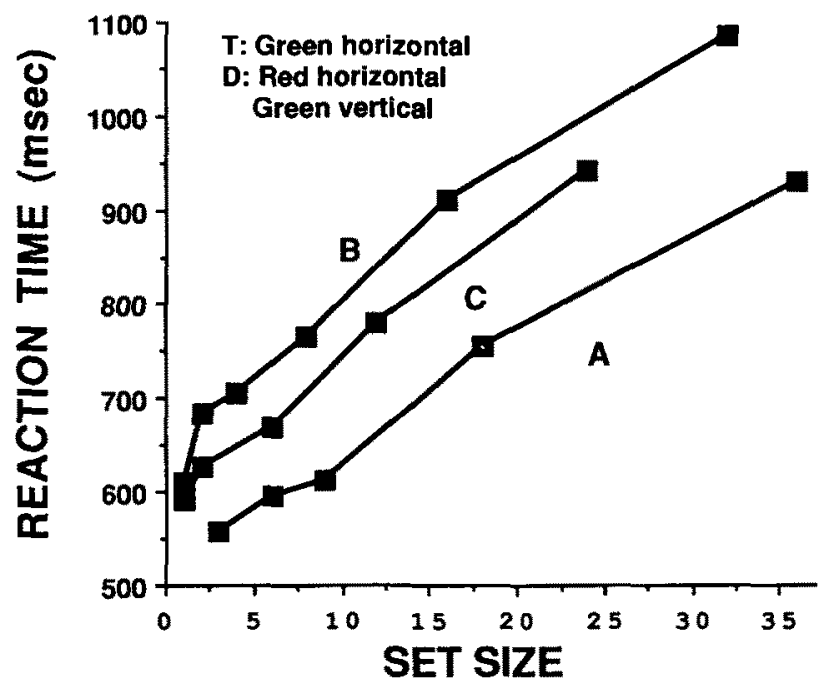

Figure 1. Average RTs as a function of set size in a search for a conjunction of color and orientation. (There are three groups of subjects [A, B, and C]. Target items [T] and distractor items [D] are identified on the figure. IA shows target trial data only; $1 \mathrm{~B}$ shows blank trials. Note that RT increases quite slowly with set size and that functions for Groups B and C appear nonlinear. See Table 2 for slopes of these functions.)

For Group A and Set Sizes 3, 6, 9, 18, and 36, error rates are $4.8 \%, 4.6 \%, 6.2 \%, 7.8 \%$, and $8.9 \%$, respectively. For Group B and Set Sizes 1, 2, 4, 8, 16, and 32, error rates are $1.4 \%, 2.8 \%, 1.0 \%, 1.2 \%, 0.4 \%$, and $0.8 \%$, respectively. For Group $\mathrm{C}$ and Set Sizes 1, 2, 6, 12, and 24, error rates are $5.2 \%, 7.5 \%, 7.0 \%, 6.5 \%$, and $10.2 \%$, respectively. There is some evidence of a speed-accuracy trade-off, in that Version $B$ has the longest RTs, the steepest slopes, and the lowest error rates. An ANOVA shows that effects of set size on errors are insignificant for all three versions (all $p s>.1$ ). 
Table 2

Slopes for RT $\times$ Set Size Functions, for Color $\times$ Orientation Task

\begin{tabular}{ccc}
\hline Group & $\begin{array}{c}\text { Target } \\
\text { trials }\end{array}$ & $\begin{array}{c}\text { Blank } \\
\text { trials }\end{array}$ \\
\hline A & 3.9 & 11.5 \\
B & 9.6 & 15.0 \\
C & 8.1 & 14.6 \\
\hline
\end{tabular}

\section{Discussion}

The results of Experiment 2 replicate and extend the results of Experiment 1. Again, subjects can find conjunctions more efficiently than would be predicted by the standard feature integration model. The feature integration model also fails to predict the nonlinearity of the results. There are several possible interpretations of that nonlinearity. Subjects could be performing a serial search for very small set sizes but not for larger set sizes. The search (be it serial or limited-capacity, parallel) could become increasingly efficient with time. Perhaps the most likely explanation is that the nonlinearity is a side effect of the density of items in the display. Because the items are placed at random within a $6 \times 6$ array, large set sizes have more near neighbors than small set sizes. Near neighbors may facilitate a search by allowing for comparison between items and identification of isolated items. O'Connell, Treisman, and Wolfe (1988) have found small effects of density consistent with this hypothesis.

The common practice of using only three set sizes in visual search experiments of this sort makes nonlinearities hard to observe. Pashler (1987a) reports a nonlinearity in his conjunction data, albeit of a different variety. In his data, slopes are shallower over the smaller set sizes. It is unclear why our results differ. The nonlinearities are of interest here because the feature integration model predicts linear functions. However, the possibility that the nonlinearities may be explained by an orthogonal factor such as item density makes them less important than the shallow slopes.

\section{Experiment 3: Conjunctions of Color and Size}

The targets in both Experiments 1 and 2 could be considered conjunctions of color and orientation. Experiment 3 examines conjunctions of color and size.

Table 3

Slopes for $R T \times$ Set Size Functions, for Color $\times$ Orientation Task

\begin{tabular}{clcc}
\hline Group & Set sizes & $\begin{array}{c}\text { Target trial } \\
\text { slopes }\end{array}$ & $\begin{array}{c}\text { Blank trial } \\
\text { slopes }\end{array}$ \\
\hline A & $3,6,9$ & 7.4 & 9.2 \\
& $9,18,36$ & 3.1 & 11.5 \\
B & $1,2,4$ & 31.5 & 28.7 \\
& $8,16,32$ & 5.3 & 13.6 \\
C & $1,2,6$ & 23.4 & 14.6 \\
& $6,12,24$ & 6.1 & 14.3 \\
\hline
\end{tabular}

Note. Slopes are computed separately for the upper and lower halves of the functions.

\section{Method}

Five subjects (drawn from the MIT undergraduate subject pool) were tested with a small ( 30 minutes of arc on a side) red square as the target and small green squares and large (50 minutes of arc on a side) red squares as distractors. Subjects ran 20 practice trials and 300 data trials. All other aspects of the experiment were the same as in Experiments 1 and 2.

\section{Results and Discussion}

Table 4 shows blank and target trial slopes for the five subjects tested with a small red square as the target and small green and large red squares as distractors. The results are comparable to those obtained for Color $\times$ Form and Color $x$ Orientation searches in Experiments 1 and 2. They are also similar to those reported by Treisman in her more recent work (Treisman, 1988). Even with a small number of subjects, it is clear that for some subjects, RTs for size-color conjunction searches are virtually independent of set size. This is true even when the target is the less salient small box.

\section{Experiment 4: Searches for Ts Among $L s$}

Conjunctions between basic features are not the only stimuli that traditionally give rise to steep slopes in visual search tasks. For example, searching for a letter $T$ among $L s$ produces results characteristic of serial search as long as the $T$ s and $L s$ are presented in a variety of orientations (Bergen \& Julesz, 1983). In attempting to understand the results of Experiments 1 and 2, it would be useful to know if our subjects are similarly efficient for all searches.

\section{Method}

The target stimulus for this experiment was a $T$ that could be presented in any of four rotations $\left(0^{\circ}, 90^{\circ}, 180^{\circ}\right.$, or $\left.270^{\circ}\right)$. The distractors were $L s$ in any of the same four rotations. Two groups of subjects from the undergraduate subject pool were used. In Group A, 9 subjects were tested on Set Sizes 8, 16, and 32. In Group B, 6 subjects were tested on Set Sizes 1, 2, 6, 12, and 24. In Group A, subjects were given 20 practice and 200 data trials. In Group B, they were given 20 practice and 500 data trials. In all other respects, the conditions of this experiment were the same as those in the previous experiments.

\section{Results and Discussion}

Figure 2 shows average RT $\times$ Set Size functions for both groups. These results fit the pattern of a serial, self-terminating search. In both groups there are strong linear trends in the target trials [Version $1, F(1,20)=33.9$; Version $2, F(1,16)$ $=82.1 ; p<.001$ for both] and in the blank trials [Version 1 , $F(1,20)=45.5$; Version $2, F(1,16)=53.1 ; p<.001$ for both]. Both groups show much steeper slopes than groups showed in the previous experiments, with target trial slopes of 24.9 and 19.2, and blank trial slopes of 60.1 and 41.6. In both cases, the target and blank slopes are significantly different [Version $1, F(1,20)=43.0$; Version $2, F(1,16)=30.8$; $p<.001$ for both]. Group A shows no hint of a quadratic 
Table 4

Color $\times$ Size Conjunctions: Individual Slopes

\begin{tabular}{ccc}
\hline Subject & $\begin{array}{c}\text { Target trial } \\
\text { slopes }\end{array}$ & $\begin{array}{c}\text { Blank trial } \\
\text { slopes }\end{array}$ \\
\hline JMW & 2.6 & 5.5 \\
SLF & 11.3 & 16.7 \\
ECB & 2.0 & 8.4 \\
JHD & 5.7 & 9.2 \\
ADP & 2.1 & 21.9 \\
\hline
\end{tabular}

trend $(F<1)$, and although the trend in Group B is in the right direction, it is far from significant, $F(1,16)=2.0$, $p>.1$.

The mean slope ratios for the two versions are 3.2 and 2.2. Neither is significantly different from $2: 1$ [Version $1, t(5)=$ 1.17 ; Version $2, t(8)=0.96 ; p>.1$ for both]. Without more subjects, however, concluding that the slope ratios are 2:1 would be unwise.

For Group A and Set Sizes 8, 16, and 32, error rates are $1.7 \%, 3.7 \%$, and $1.2 \%$, respectively. For Group B and Set Sizes $1,2,4,8,16$, and 32 , error rates are $1.2 \%, 2.2 \%, 2.3 \%$, $2.9 \%, 4.9 \%$, and $11.2 \%$, respectively. Subjects in Group B were also run as Group B in Experiment 2. Error rates are higher for the $T$ versus $L$ task than for the conjunction task. This contradicts the hypothesis that shallow slopes in the conjunction task are obtained by sacrificing accuracy.

Whereas the results from the conjunction experiments $(1$, 2 , and 3 ) differ strikingly from the usual pattern for serial, self-terminating search, in Experiment 4 , searches for $T \mathrm{~s}$ among $L s$ show the same steep, linear RT $\times$ Set Size functions seen in other studies. Thus, it is the results for conjunctions of two features that require explanation.

\section{Search for T among Ls}

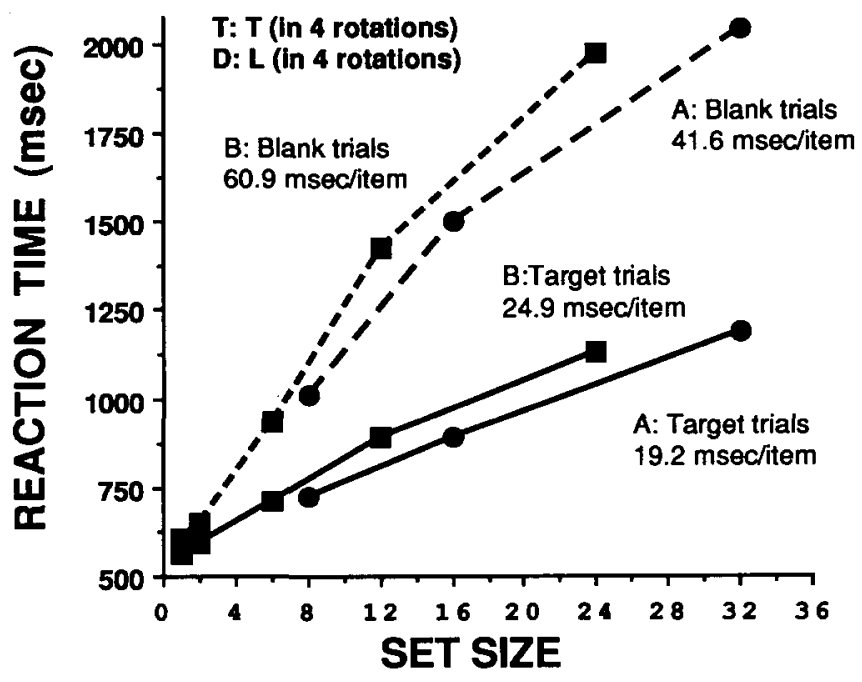

Figure 2. Searches for a $T$ among $L$ s. (Average data are given for two groups of subjects [A and B]. The results are consistent with serial, self-terminating search. Target items [T] and distractor items [D] are identified on the figure.)

\section{Experiment 5: Practice Effects in Conjunction Tasks}

Some search tasks exhibit practice effects. For example, Schneider and Eberts (1980) ran subjects for thousands of trials and found that RT $\times$ Set Size slopes declined somewhat, but never to less than $10 \mathrm{~ms} /$ item for target trials and about twice that for blank trials. In contrast, no subject in our Experiment 1 had more than 20 trials of practice in conjunction experiments or more than 60 trials of total practice in the visual search paradigm. Nevertheless, 16 of these 20 unpracticed subjects in Experiment 1 had target trial slopes under $10 \mathrm{~ms} / \mathrm{item}$. Thus, any practice effect explanation for our results would have to invoke transfer of some form of practice outside of our particular experimental paradigm. Many of our subjects were drawn from the MIT subject pool and might have had experience in other RT experiments. In Experiments 5 and 6, we examined the effects of practice in our paradigm.

\section{Method}

Five naive, unpracticed undergraduate subjects were tested on a replication of Experiment 1 . The target was a red $O$. The distractors were red $X$ s and green $O$ s. Set sizes were 8, 16, and 32. Each subject was run on five blocks of 220 trials. Blocks were run on consecutive days. The first 20 trials in each block were practice. The remaining 200 were used to obtain RT $\times$ Set Size slopes for target and blank trial conditions. In all other respects, the experiment was identical to Experiment 1.

\section{Results and Discussion}

Slopes for target and blank trials for the five blocks for each subject are shown in Table 5.

Separate ANOVAs for target and blank trials reveal significant effects of practice for blank trials, $F(4,16)=4.7, p=.011$, but not for target trials, $F(4,16)=1.9, p>.15$. The effect is probably not seen in the target trials because of a "floor effect." For 4 of the 5 subjects, slopes were less than $10 \mathrm{~ms}$ on the first block. As in the preceding conjunction experiments, most of our subjects start with very shallow slopes. It is, therefore, no great surprise that they do not show much improvement. Even the $y$-intercepts of the RT $\times$ Set Size functions do not decrease much with practice in this experiment. Subject LCY does change from what appears to be a serial, self-terminating search to the more efficient search performed by our other subjects during the course of this experiment.

\section{Experiment 6: Practice Effects on Searches for Ts Among $L s$}

\section{Method}

In Experiment 4, we obtained steeper slopes with a search for $T \mathrm{~s}$ among $L s$. There is, therefore, more room for improvement in this task. To test for practice effects, 5 subjects repeated Group A of Experiment 3 (Set Sizes 8, 16, and 32) five times (for a total of 1,100 trials). The stimuli were red rather than white. In all other respects 
Table 5

Practice Effects: Color $\times$ Form Conjunctions, Slopes for Target and Blank Trials

\begin{tabular}{|c|c|c|c|c|c|c|c|c|c|c|}
\hline \multirow[b]{2}{*}{ Block } & \multicolumn{2}{|c|}{ RS } & \multicolumn{2}{|c|}{ LCY } & \multicolumn{2}{|c|}{ JD } & \multicolumn{2}{|c|}{ DB } & \multicolumn{2}{|c|}{ JMD } \\
\hline & Targ & Blnk & Targ & Blnk & Targ & Blnk & Targ & Blnk & Targ & Blnk \\
\hline 1 & 5.3 & 36.9 & 29.9 & 60.3 & 8.6 & 19.6 & 5.4 & 9.0 & 4.2 & 6.0 \\
\hline 2 & -0.4 & 17.8 & 13.1 & 62.3 & 4.1 & 6.7 & 4.9 & 5.8 & 4.0 & 7.4 \\
\hline 3 & 7.3 & 20.9 & 7.2 & 50.0 & 4.3 & 2.7 & 2.7 & 5.8 & 2.4 & -1.2 \\
\hline 4 & 5.3 & 16.7 & 1.9 & 58.7 & 5.0 & 6.8 & 3.1 & 6.1 & 2.4 & 1.8 \\
\hline 5 & 1.1 & 10.3 & 6.2 & 33.5 & 5.5 & 5.3 & 2.3 & 4.8 & 2.5 & 1.0 \\
\hline
\end{tabular}

Note. $\quad$ Targ $=$ target, Blnk $=$ blank.

the experiment was identical to Group A of Experiment 4. The same subjects were tested in Experiments 5 and 6.

\section{Results and Discussion}

Table 6 shows the results for this experiment. There is some improvement with practice, most of it after the first block of 220 trials. After that, slopes hover around 8-15 ms/item for target trials. Slopes for blank trials are more variable across subjects. Separate ANOVAs for target and blank trials show that the main effects of practice are significant for target trials, $F(4,16)=3.1, p<.05$, but not for blank trials $(F<1)$.

A comparison of the results of this experiment with the results for the same subjects in Experiment 5 (conjunction practice) in a single ANOVA shows that the target trial slopes are shallower in the conjunction case, $F(1,4)=16.4, p<$ .05. There is no evidence that this difference changes with practice, $F(4,16)=1.3, p>.3$.

The results of Experiments 5 and 6 reveal modest practice effects when floor effects do not interfere. These effects are not adequate to explain the differences between our data and previously published results for similar searches. Furthermore, these results confirm that our subjects do what appears to be a classic, serial, self-terminating search for a $T$ among $L s$, but do something quite different for conjunctions of color and form.

\section{Experiment 7: Stimulus Salience}

In our experiments, we use saturated red and green stimuli on a black background. Treisman's earlier work involved less striking stimulus colors and a white background. She suggests that the differences between her results and those presented here are explainable by these differences in stimulus salience (A. Treisman, personal communication). Indeed, she has shown that the less salient the perceptual distinction between target and distractors, the steeper the resulting RT $\times$ Set Size functions (Treisman \& Gormican, 1988). It is important to remember, however, that even if stimulus salience explains the difference between our results and previously published work, our results would still raise doubts about the standard feature integration model, because our results show that searches for conjunctions of color and form can yield very shallow slopes. In Experiment 7, we attempted to emulate Treisman's stimuli in our computer display.

\section{Method}

In this experiment, subjects searched for a red, left, oblique line among green, left, obliques and red, right, obliques. Treisman's original stimuli (Treisman \& Gelade, 1980) were drawn in ink on white cards for presentation in a tachistoscope. To emulate her situation, the red and green used here were less saturated then the stimuli in our previous experiments (CIE $x, y$ coordinates: red, .42 , .35 ; green, $.32, .41$; luminance, .86 and $\left..50 \mathrm{~cd} / \mathrm{m}^{2}\right)$. These stimuli were presented on a brighter white background (CIE $=.34, y=.34$, luminance $=1.5 \mathrm{~cd} / \mathrm{m}^{2}$ ). Oblique stimuli were used because the difference between left and right oblique is less salient than that between vertical and horizontal, and because Treisman (Treisman \& Gelade, 1980) used oblique lines. As a control, subjects were also tested with the same oblique stimuli using the saturated reds and greens on a black background that we used in Experiments 1-6.

Ten subjects from the undergraduate subject pool were tested for 330 trials each. The first 30 trials were discarded as practice. Set sizes of 8,16 , and 32 elements were used. In all other respects the method was identical to that of Experiment 2.

\section{Results}

Average results for target and blank trials from the two conditions are shown in Figure 3. The emulation of Treis-

Table 6

Practice Effects: $T$ Versus L Searches

\begin{tabular}{|c|c|c|c|c|c|c|c|c|c|c|}
\hline \multirow[b]{2}{*}{ Block } & \multicolumn{2}{|c|}{$\mathbf{R S}$} & \multicolumn{2}{|c|}{ LCY } & \multicolumn{2}{|c|}{ JD } & \multicolumn{2}{|c|}{ DB } & \multicolumn{2}{|c|}{ JMD } \\
\hline & Targ & Blnk & Targ & Blnk & Targ & Blnk & Targ & Blnk & Targ & Blnk \\
\hline 1 & 23.5 & 62.3 & 28.5 & 59.5 & 26.0 & 55.8 & 8.3 & 22.6 & 14.2 & 20.4 \\
\hline 2 & 13.0 & 61.8 & 16.3 & 77.0 & 8.5 & 23.5 & 15.3 & 18.1 & 7.5 & 20.3 \\
\hline 3 & 31.6 & 98.7 & 20.2 & 60.7 & 9.5 & 11.8 & 17.3 & 26.0 & 8.5 & 22.3 \\
\hline 4 & 22.5 & 54.5 & 14.8 & 73.7 & 7.8 & 12.3 & 11.9 & 24.5 & 11.6 & 15.3 \\
\hline 5 & 18.0 & 74.7 & 9.8 & 40.8 & 12.0 & 20.6 & 7.7 & 13.3 & 2.5 & 1.0 \\
\hline
\end{tabular}

Note. Targ = target, Blnk = blank. 


\section{Tachistoscope Emulation}

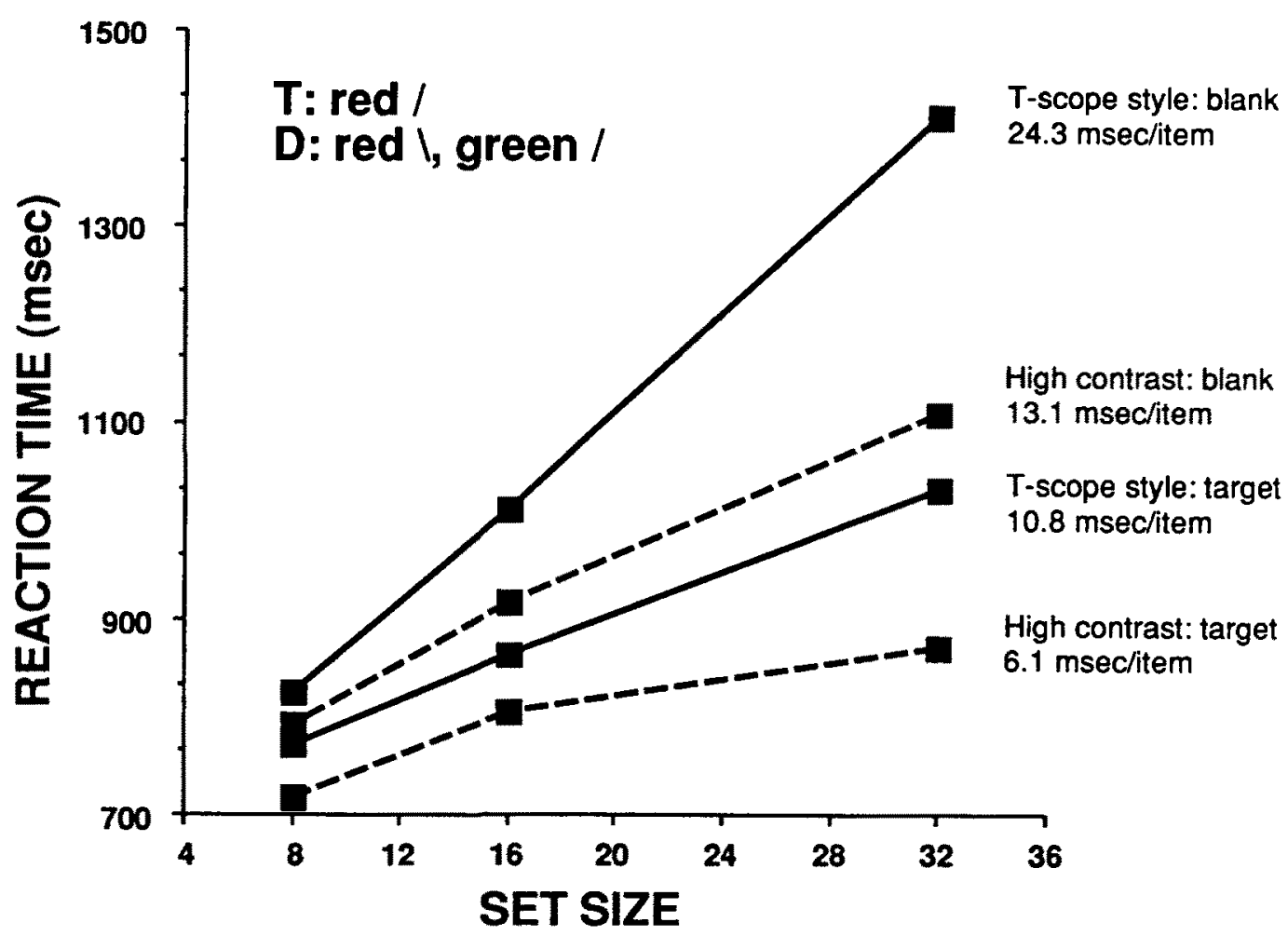

Figure 3. Average RTs as a function of set size in a search for a conjunction of color and orientation. (In the "high contrast" condition, saturated color stimuli are presented on a dark background. In the "T-scope" condition, less saturated colors are presented on a bright background, emulating the ink-onpaper conditions of Treisman and Gelade [1980]. Target items [T] and distractor items [D] are identified on the figure.)

man's (Treisman \& Gelade, 1980) conditions (labeled T-scope style) produces steeper slopes than does the control condition (labeled High contrast). The two conditions of this experiment produce significantly different slopes of RT $\times$ Set Size functions, paired $t$ test: target trial slopes, $t(9)=6.3, p<.001$; blank trial slopes, $t(9)=2.8, p<.05$.

The increase in RT with set size is significant in the Tscope emulation for target and for blank trials, $F(1,18)=$ $20.0, p<.001 ; F(1,18)=23.3, p<.001$. The increase is significant in the control condition as well, $F(1,18)=46.6$, $p<.001 ; F(1,18)=57.7, p<.001$. The $y$-intercepts are similar in the two conditions (see Figure 3). For both conditions of this experiment, the interaction of set size and trial type is significant, T-scope: $F(2,18)=4.4, p<.05$; control: $F(2,18)=18.2, p<.001$.

Serial, self-terminating search predicts a $2: 1$ ratio between the slopes for blank and target trials. Slope ratios were computed for each subject. In the T-scope emulation, the average ratio is 2.4 with a range of $0.8-3.9$. By contrast, the average slope ratio in the control condition is higher (3.5) with a much greater range, 0.4-13.7. Neither average ratio is significantly different from 2.0 , T-scope: $t(9)=1.5, p>.05$; control: $t(9)$ $=1.8, p>.1$.
If the individual slopes for the blank trials are plotted as function of the slopes for the target trials, the $2: 1$ slope ratio predicted by a serial, self-terminating search predicts a linear function with a slope of 2.0 . The relationship of blank trial slopes to target trial slopes has a significant linear component for the T-scope emulation $\left(r^{2}=.71, p<.05\right)$ and the slope of the best-fit line is 1.8 (close to 2.0). For the control condition, the linear relationship does not hold $\left(r^{2}=.27, p>.1\right)$ and the slope of the best fit line is 1.1 . The results of the T-scope emulation are in better agreement with the predictions of the feature integration model than the results of the control condition.

Average error rates for the T-scope style were $4.6 \%$ for Set Sizes 8 and 16 , and $8.1 \%$ for Set Size 32. The control condition had lower error rates at all set sizes $(4.4 \%, 3.9 \%$, and $5.1 \%$ ).

\section{Discussion}

The emulation of Treisman's original (Treisman \& Gelade, 1980 ) conditions produced results quite consistent with serial, self-terminating search. The slopes are steeper than those in Experiments 1-6, though not as steep as those reported in 
Treisman and Gelade (1980). The slopes are comparable to those obtained by Egeth, Virzi, and Garbart (1984) in a similar task. In Experiments 1-6, there was little evidence of a reliable 2:1 slope ratio between blank and target trials. In the T-scope emulation, that evidence is quite strong.

The similarity to the results of Egeth et al. (1984) suggest that one of the two stimulus dimensions was still salient. In Experiment 8 we attempted to further reduce the salience of both orientation and color.

\section{Experiment 8}

\section{Method}

The target was a blue left oblique line. Distractors were red oblique, green oblique, blue vertical, and blue horizontal lines in equal proportion. This task was made more difficult than Experiments 1-7 by increasing the number of different colors and different orientations from 2 to 3 and by decreasing the difference between colors and orientations. In particular, the blue and green used were not as strikingly different as the red and green.

Ten subjects from the undergraduate subject pool ran 330 trials evenly divided between target and blank conditions and between set sizes of 16, 24, and 32 items. The first 30 trials were discarded as practice.

\section{Results}

As shown in Figure 4, average slopes were $25.9 \mathrm{~ms} /$ item for target trials and $43.3 \mathrm{~ms} /$ item for blank trials. RT increases linearly with set size, target trials: $F(1,18)=90.3, p<.001$;

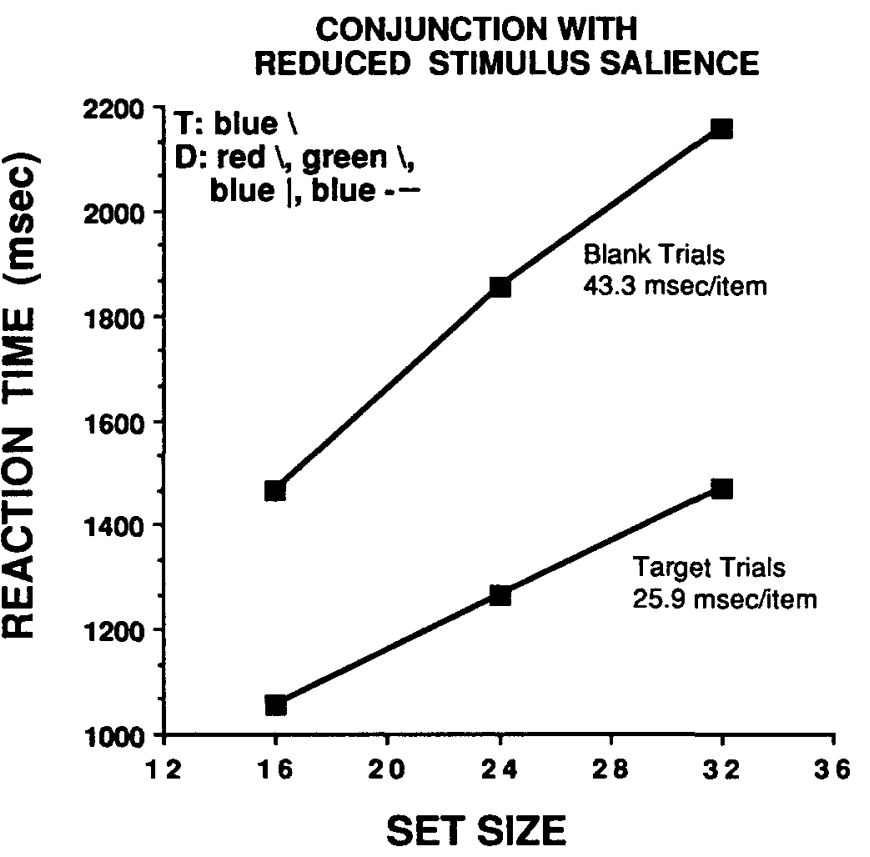

Figure 4. Average RTs as a function of set size in a search for a conjunction of color and orientation in low salience conditions. (Results are consistent with serial, self-terminating search. Target items [T] and distractor items [D] are identified on the figure.) blank trials: $F(1,18)=77.7, p<.001$, and the two slopes differ significantly, $F(1,18)=14.2, p<.005$. The average slope ratio is 1.8 . This does not differ significantly from 2.0 , paired $t$ test, $t(9)=.88, p>.4$. Error rates are quite high, averaging $7.6 \%$ for Set Size $16,10.6 \%$ for set size 24 , and $14.2 \%$ for Set Size 32 .

These results essentially replicate Treisman's older (Treisman \& Gelade, 1980) results. The slopes are comparable and appear to be in the 2:1 ratio predicted by serial, self-terminating search. The error rates are higher than in the shallow slope conjunction search, indicating again that the shallow slopes were not obtained by sacrificing accuracy.

It seems likely that differences in stimulus salience between the experiments explain differences between our results and previously published results. The fact that stimulus salience plays an important role in determining whether the search for conjunctions is serial or not is useful in developing a new model. We will return to this point below.

\section{Discussion of Experiments 1-8}

Several conclusions may be drawn from the first eight experiments:

1. In these experiments, results of visual search for conjunctions of Color $\times$ Form, Color $\times$ Orientation, and Color $\times$ Size do not conform to the predictions of the standard feature integration model. Indeed, many subjects, including naive, unpracticed subjects, produce RTs that are nearly independent of set size.

2. The differences between these results and previously published results cannot be explained as reflecting a general ability on the part of our subjects to do all search tasks in parallel, because searches for $T$ s among $L s$ are serial.

3. It is unlikely that the differences are due to practice effects.

4. Differences in stimulus salience appear to account for a substantial portion of the differences in results.

The central fact is that the data from these conjunction experiments violate the predictions of the standard feature integration model. Indeed, in light of the present results, the recent spate of shallow $\mathrm{RT} \times$ Set Size functions in conjunction experiments may be seen as the rule in such searches and not merely as an accumulation of exceptions (Egeth et al., 1984; Nakayama \& Silverman, 1986; Pashler, 1987b; Steinman, 1987; Treisman, 1988). We now turn to a modification of the feature integration model that can explain these and other results. Here we will present a qualitative description of our modified model. A more formal presentation with a computer simulation of the results of our experiments and those of other researchers can be found elsewhere (Cave \& Wolfe, 1988).

\section{The Guided Search Model}

As noted in the introduction, a curious characteristic of the standard feature integration model is that the model holds that the parallel and serial processes are autonomous. The serial process cannot use information collected by the parallel process. If it could, there would be no need for a complete 
serial, self-terminating search for conjunctions. Although a parallel feature map cannot pinpoint the location of a conjunction target, it can divide the set of all items into those that could be the target and those that could not. To return to the example in the introduction, if the target is a red $X$, a parallel color map can divide items into red and green items. Any one of the red items could be the target if it were also an $X$, whereas none of the green items could be the target. Similarly, a form (or orientation) map could divide the items into $X s$ (any one of which could be the target if it were also red) and $O$ s (none of which could be the target).

One can easily imagine a mechanism that would allow the serial stage to take advantage of this ability of the parallel maps to divide items into distractors and candidate targets. Suppose that each parallel feature map excites all of the spatial locations of candidate targets in a map that embodies the serial, attentional stage of processing. All of the red locations will be excited, all of the $X$ locations will be excited, and the "red $X^{\prime \prime}$ location (if any) will be doubly excited. If the "spotlight of attention" is directed to the point of maximum excitation, it will find the target without the necessity of conducting a random, serial search. This is illustrated in Figure 5.

This model works equally well if we assume that the parallel processes inhibit nontarget locations, discarding distractors rather than identifying candidate targets. Indeed, these two versions of the guided search model are very hard to distinguish from each other. This topic will be considered in a separate article (Cave \& Wolfe, in press). Either version implies that top down instructions about the nature of the target can reach the parallel processes or, at least, can gate the output of those processes.

If the transmission of information from the parallel to the serial stage were perfect, attention would be guided immediately to the target, and conjunctive searches would be independent of set size. Our data suggest that the transmission of

\section{Standard Conjunction (color $\mathrm{x}$ orientation) Target Distractors: $=$ ]}

Stimulus

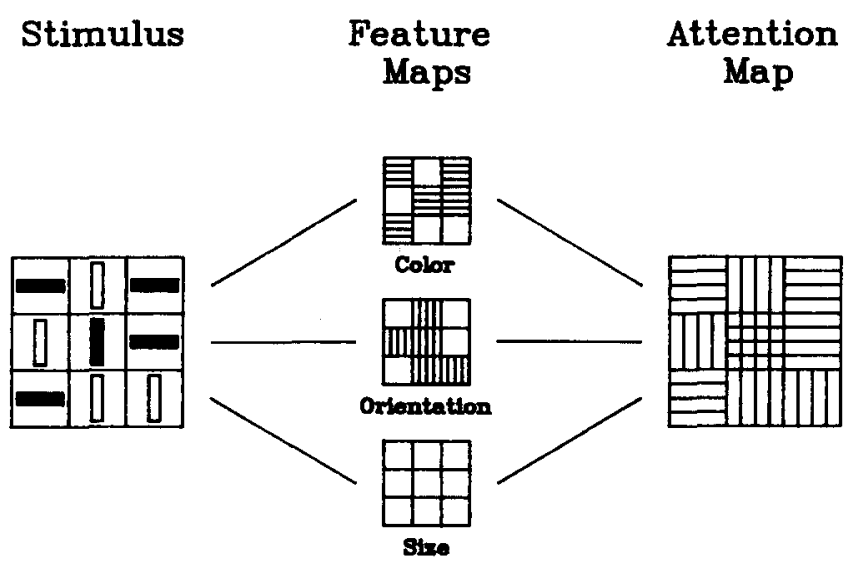

Figure 5. The guided search model for standard conjunction searches. (See text for details.) information is imperfect. The efficiency of the search is improved over a random, serial search, but it appears that the serial mechanism still examines some incorrect items. One way to model this imperfect transmission is to assume that there is noise in the signal from the parallel to the serial stage. If the noise is substantial, the parallel stage is of no use to the serial stage, and the search will be strictly serial. If there is minimal noise, the serial stage should be able to locate a target without examining any incorrect items. Considered in these terms, the differences between subjects and, indeed, between laboratories, can be modeled as variations of a single noise variable. Variation of stimulus salience (Experiment 7) can be seen as a manipulation of the signal to noise ratio in this task. As noted in the introduction, this model is very similar in its outlines to a model proposed by Hoffman $(1978,1979)$, though his model was not applied to searches for conjunctions.

Visual search takes place over time. During the time that the search is occurring, the information from the parallel processes to the serial process can be updated. With continuously visible stimuli, there is no reason to assume that the parallel processes analyze the stimulus, pass their findings on to the serial process, and then shut down. The parallel processes must continue to "see" the stimuli, just as do the i photoreceptors or the ganglion cells of the retina. The noise in the transmission from parallel to serial stage is random; the signal is not. Therefore, the parallel processes might incorrectly guide the spotlight to a distractor at first. However, with the passage of time, the signal should emerge from the noise to guide the spotlight of attention to the target. We are proposing that the search for conjunctions is serial, but not random. Each time the spotlight of attention is moved, it is directed to the most likely target location, as identified by the parallel processes. Continuous updating of the input from the parallel processes makes it more and more likely that those processes will correctly identify the target location as the most likely location. Under some conditions, this accumulation of information could yield nonlinear slopes of the sort seen in Experiment 2.

The same model can be applied to feature searches. In fact, in this model, there is no qualitative difference between searches for basic features and searches for conjunctions. In both cases, the parallel processes provide the signal to guide attention to the target. In a simple feature search, the presence of a unique feature (e.g., red among green) generates a strong signal that quickly exceeds the background noise. As stimulus salience is reduced, the signal indicating the presence of a unique feature is hidden by the noise. In this case, the serial process would examine more of the distractors before the signal from the appropriate feature map exceeded the noise in that map and guided the serial process to the target location (see Treisman \& Gormican, 1988; note also that Treisman \& Gelade, 1980, obtained small positive slopes for their feature searches).

This strategy of using the parallel processes to help the serial process locate likely targets will not work for all search tasks. Consider the $T$ versus $L$ task (Experiment 4 ). In this case, both the target $T$ and the distracting $L s$ are made up of a vertical and a horizontal line. As illustrated in Figure 6, a 
Search for T among L's

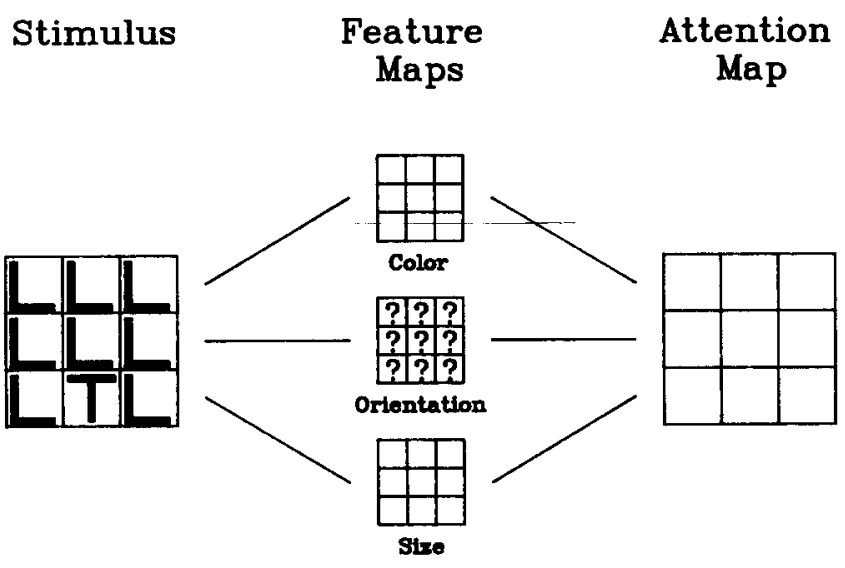

Figure 6. The guided search model for $T$ versus $L$ searches. (See text for details.)

feature map sensitive to orientation would "see" the same features at all locations. Even over time, the feature map would be unable to guide the attentional spotlight, and resulting searches would be serial, as they are in Experiment 4 .

The guided search model makes predictions that are qualitatively different from those of the standard feature integration model. Under the guided search model, one would expect more efficient searches if more information from the parallel processes could be used by the serial process. In a conjunction experiment, each distractor item has one feature that eliminates it as a candidate target (e.g., wrong color or wrong orientation). By conjoining three features (e.g., Color $\times$ Orientation $\times$ Size), we can arrange for each distractor to possess two features that eliminate it as a candidate target. If two sources of information are better than one, as the guided search model predicts, then these triple conjunctions will produce more efficient searches than standard conjunctions.

\section{Experiment 9: Triple Conjunctions}

In this experiment, subjects searched for targets defined by a triple conjunction of color, form, and size. The logic of this experiment is illustrated in Figure 7. Consider a big, black, vertical line as the target. In a triple conjunction task, distractors can share zero, one, or two features with the target. In this example, all distractors share one feature with the target. This yields as a distractor set, (a) big, white, horizontal, (b) small, black, horizontal, and (c) small, white, vertical. In terms of the guided search model, there are now three sources of information that can be used to constrain the movements of the spotlight of attention. In a simple conjunction, there are only two. As noted above, this leads to the prediction that searches for triple conjunctions should be more efficient than searches for simple conjunctions. The standard feature integration model would not make this prediction. If the act of conjunction by attention can be performed as efficiently across three features as across two, then the standard feature integration model would predict that the slopes for triple conjunctions would be about the same as those for simple conjunctions. If it takes longer to determine that an item is vertical, white, and large than it does to determine merely that it is vertical and white, then the standard model predicts that slopes for triple conjunctions would be steeper than those for simple conjunctions.

Quinlan and Humphreys (1987) did an experiment of this sort and found that triple conjunctions produced shallower slopes than simple conjunctions when the target shared only one feature with each distractor. The slopes for their triple conjunction searches were quite steep by our standards, averaging $11.6 \mathrm{~ms} /$ item on target trials and $28.6 \mathrm{~ms} /$ item on blank trials (Quinlan \& Humphreys, 1987; Table 8). In our replication of this experiment, RT is virtually independent of set size.

In a triple conjunction where targets and distractors share two features, there is only one piece of information that distinguishes targets and distractors. In this case, there is no more information in the triple conjunction than in the standard conjunction. The guided search model would predict that search times would be similar in these two cases. Quinlan and Humphrey's (1987) data support this hypothesis.

\section{Method}

Eleven subjects from the undergraduate subject pool were tested in each of four conditions. Conditions 1 and 2 were triple conjunction tasks where the distractors shared one feature with the target. In Condition 1, the target was big. In Condition 2, the target was small. Condition 3 was a triple conjunction task where the distractors each shared two features with the target. Finally, Condition 4 was a simple conjunction task replicating Experiment 1 . The stimuli for the four conditions are described in Table 7.

\section{Triple Conjunction}

Target: | Distractors: - $\square \square$

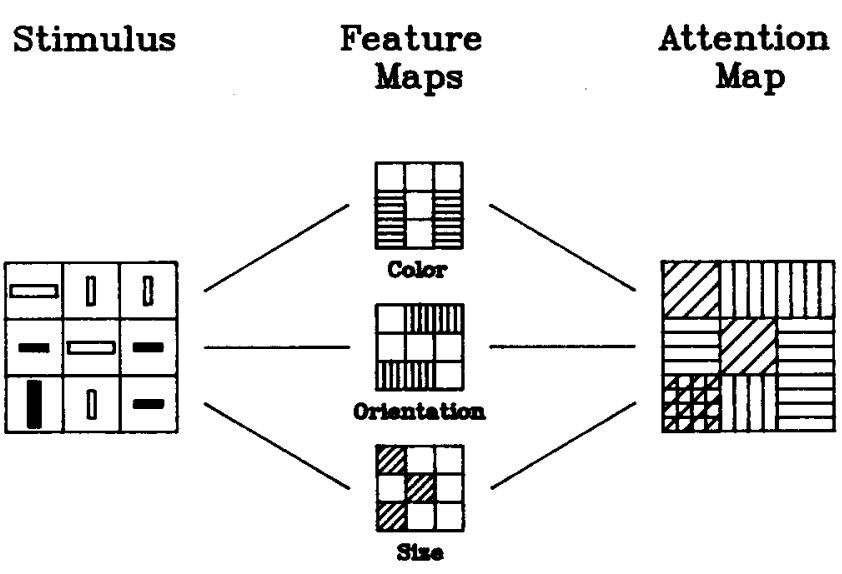

Figure 7. The guided search model for triple conjunction searches. (See text for details.) 
Table 7

Stimuli for Experiment 9: Triple Conjunctions

\begin{tabular}{|c|c|c|c|}
\hline Condition & Target & Distractors & Task \\
\hline 1 & Big red $O$ & $\begin{array}{l}\text { Small red } X \\
\text { Small green } O \\
\text { Big green } X\end{array}$ & $\begin{array}{l}\text { Triple conjunction } \\
\text { sharing } 1 \text { feature }\end{array}$ \\
\hline 2 & Small red $X$ & $\begin{array}{l}\text { Big green } X \\
\text { Big red } O \\
\text { Small green } O\end{array}$ & $\begin{array}{l}\text { Triple conjunction } \\
\text { sharing } 1 \text { feature }\end{array}$ \\
\hline 3 & Big red $O$ & $\begin{array}{l}\text { Big red } X \\
\text { Big green } O \\
\text { Small red } O\end{array}$ & $\begin{array}{l}\text { Triple conjunction } \\
\text { sharing } 2 \text { features }\end{array}$ \\
\hline 4 & Big red $O$ & $\begin{array}{l}\text { Big red } X \\
\text { Big green } O\end{array}$ & Simple conjunction \\
\hline
\end{tabular}

All distractor items were presented with equal probability. Set sizes of 8,16 , and 32 were used. The order of conditions was random across subjects. In each condition, the subject had 30 practice trials followed by 300 data trials. Targets were presented on $50 \%$ of trials.

\section{Results}

Slopes for individual subjects and averages of those slopes are shown in Table 8 for all four conditions. Average RTs and $y$-intercepts are comparable to those in Experiments 1-8 (see Figures 8-10). The main effect of set size is insignificant in Condition 1, $F(2,20)=2.1$ (target), 2.3 (blank); $p>.1$ in both cases. For this condition, RT is independent of set size. The main effects of set size are significant for all other conditions.

The results are best understood when compared in pairs. When comparing slopes in the statistical analyses, we included target trials only, and used contrasts from an ANOVA with subject, set size, and condition as variables. When testing overall differences between conditions, we included both target and blank trials, and used contrasts from an ANOVA with subject, set size, condition, and target presence as variables.
Figure 8 shows average RTs for target and blank trials for Conditions 1 and 4, comparing a one-shared-feature triple conjunction search to a standard conjunction search for the same target. Overall, triple conjunction responses are faster than standard conjunction responses, $F(1,30)=26.7, p<$ .001 , and practically independent of the number of distractors. The triple conjunction slopes are very shallow; $0.4 \mathrm{~ms} / \mathrm{item}$ for target trials and $1.2 \mathrm{~ms} /$ item for blank trials. This is less than the standard conjunction slope of $5.2 \mathrm{~ms} / \mathrm{item}$ for the target trials and $10.0 \mathrm{~ms} /$ item for the blank trials. The difference is significant, as shown by comparing linear contrasts for the target trials, $F(1,60)=28.1, p<.001$. Note that the standard conjunction results are shallow and appear to be quite serial only because of the scale used in Figure 8.

Figure 9 compares the simple conjunction results of Condition 4 with the triple conjunction search in Condition 3 , in which each distractor shared two features with the target. There is no overall difference between these two conditions $(F<1)$ and there is no difference between the slopes $(F<1)$. When triple conjunction distractors differ from the target in only one feature, a search is no easier than it is in the standard conjunction task.

A triple conjunction search is significantly faster when distractors share only one feature with the target (Condition 1) than when they share two (Condition 3 ), $F(1,30)=23.5$, $p<.001$. Slopes are significantly less in Condition 1 as well, $F(1,60)=20.1, p<.001$. Subjects do better at searches with one-shared-feature triple conjunctions than with either standard conjunctions or two-shared-feature triple conjunctions, even when the target is the same in each case.

Figure 10 compares two types of one-shared-feature triple conjunction search. The target is big in Condition 1 and small in Condition 2. When the target is small, responses are slower, $F(1,30)=5.0, p<.05$, and positive slopes are steeper, $F(1$, $60)=5.9, p<.025$. When the target is big, the slope of the average data is $0.4 \mathrm{~ms} /$ item for target trials and $1.2 \mathrm{~ms} / \mathrm{item}$ for blank trials. When the target is small, those slopes increase to 2.6 and $5.5 \mathrm{~ms} /$ item, respectively.

Error rates for Set Sizes 8, 16, and 32 are 1.7\%, 2.3\%, and $0.7 \%$ in Condition $1 ; 2.6 \%, 3.2 \%$, and $3.8 \%$ in Condition 2;

Table 8

Individual Slopes for Subjects in Four Conditions of Experiment 8

\begin{tabular}{|c|c|c|c|c|c|c|c|c|}
\hline \multirow[b]{2}{*}{ Subject } & \multicolumn{2}{|c|}{ Condition 1} & \multicolumn{2}{|c|}{ Condition 2} & \multicolumn{2}{|c|}{ Condition 3} & \multicolumn{2}{|c|}{ Condition 4} \\
\hline & Yes & No & Yes & No & Yes & No & Yes & No \\
\hline $\mathrm{AC}$ & -0.6 & 1.5 & 2.3 & 5.6 & 5.1 & 19.9 & 3.4 & 9.2 \\
\hline ADP & 0.7 & 1.5 & 2.8 & 2.0 & 2.9 & 3.2 & 3.6 & 5.4 \\
\hline BJM & -1.2 & 1.5 & 2.3 & 6.1 & 2.1 & 8.0 & 8.5 & 12.9 \\
\hline CLT & 1.1 & 0.7 & 1.5 & 3.3 & 2.8 & 3.6 & 1.8 & 3.7 \\
\hline DBZ & 1.9 & 7.4 & 2.6 & 9.9 & 9.4 & 11.9 & 4.6 & 7.8 \\
\hline DVP & 0.5 & 0.6 & 1.8 & 2.9 & 1.5 & 4.1 & 0.2 & 3.0 \\
\hline KPY & 0.4 & 1.1 & 3.9 & 4.8 & 3.4 & 8.1 & 2.9 & 8.3 \\
\hline MHK & 1.1 & 0.6 & 4.3 & 11.1 & 4.8 & 11.2 & 18.3 & 30.2 \\
\hline RES & 0.7 & 0.7 & 4.5 & 9.4 & 0.0 & 7.9 & 10.2 & 18.1 \\
\hline SWD & -0.2 & -2.6 & 2.6 & 1.6 & 5.9 & 6.8 & 2.1 & 8.8 \\
\hline TBT & 0.8 & 0.7 & 0.4 & 3.6 & 4.7 & 1.9 & 2.1 & 2.4 \\
\hline$M$ & 0.5 & 1.2 & 2.6 & 5.5 & 3.8 & 7.9 & 5.2 & 10.0 \\
\hline$S D$ & 0.9 & 2.3 & 1.2 & 3.3 & 2.5 & 1.5 & 1.6 & 2.4 \\
\hline
\end{tabular}


$2.3 \%, 2.9 \%$, and $5.1 \%$ in Condition 3 ; and $1.7 \%, 2.8 \%$, and $5.3 \%$ in Condition 4 . These rates are quite low even though the slopes in this experiment are lower than those in any of the other experiments in this article. Indeed, the lowest slopes (Condition 1) are accompanied by the lowest error rates in this experiment. The effect of experimental condition is significant, $F(3,120)=3.7, p<.05$.

\section{Discussion}

In Condition 1 of the experiment, RT is independent of set size even though the target is defined by a conjunction, here a triple conjunction. This finding does not fit within the feature integration model, but it is accommodated by the guided search model proposed here. The shallowest slopes are obtained when each distractor differs from the target on two dimensions. In these conditions ( 1 and 2 ), more information from the parallel processes is available to guide attention than in the simple conjunction condition (4). When distractors differ from targets on only one dimension (Condition 3 ), the results are virtually identical to those obtained with simple conjunctions. As noted above, this is the predicted result under the guided search model. The two-shared-features case is equivalent to the simple conjunction case because, in both cases, the parallel processes have only one chance to discard each distractor.

In summary, the spotlight of attention can be guided by preattentive mechanisms. A parallel feature map can partition the set of items into distractors and candidate targets. This information, degraded by noise, can be passed to the serial process. In the serial process, the spotlight of attention moves from item to item until a target is found. With the aid of information from the parallel processes, however, that movement need not be random. Instead, the spotlight can be

\section{Triple Compared to Simple Conjunction}

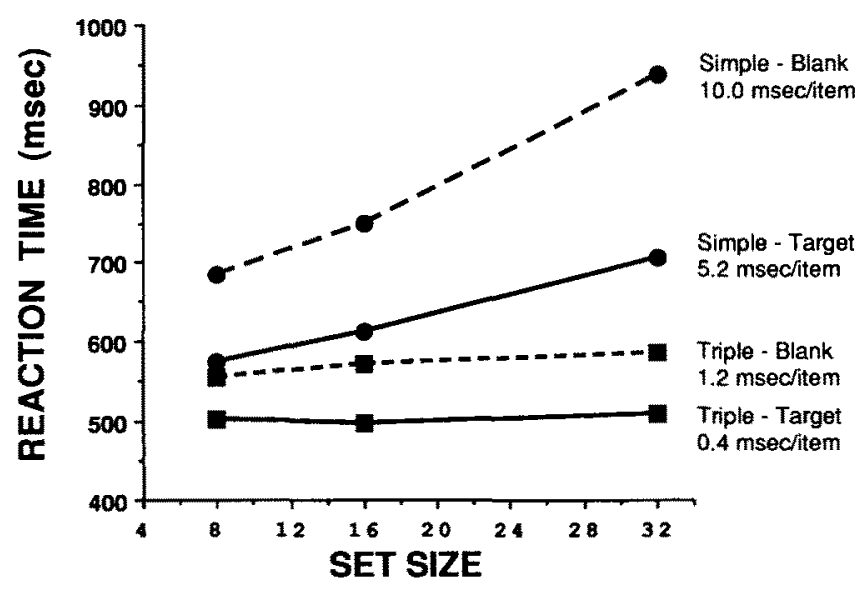

Figure 8. Comparison between results for triple and simple conjunction searches. (Average data for 11 subjects. In the triple conjunction, distractors share only one feature with the target. Slopes for triple conjunction search are $0.4 \mathrm{~ms} / \mathrm{item}$ for target trials and $1.2 \mathrm{~ms} /$ item for blank trials. Slopes for standard conjunction average $5.2 \mathrm{~ms} /$ item for target trials and $10.0 \mathrm{~ms} /$ item for blank trials.)
Triple Compared to Simple Conjunction

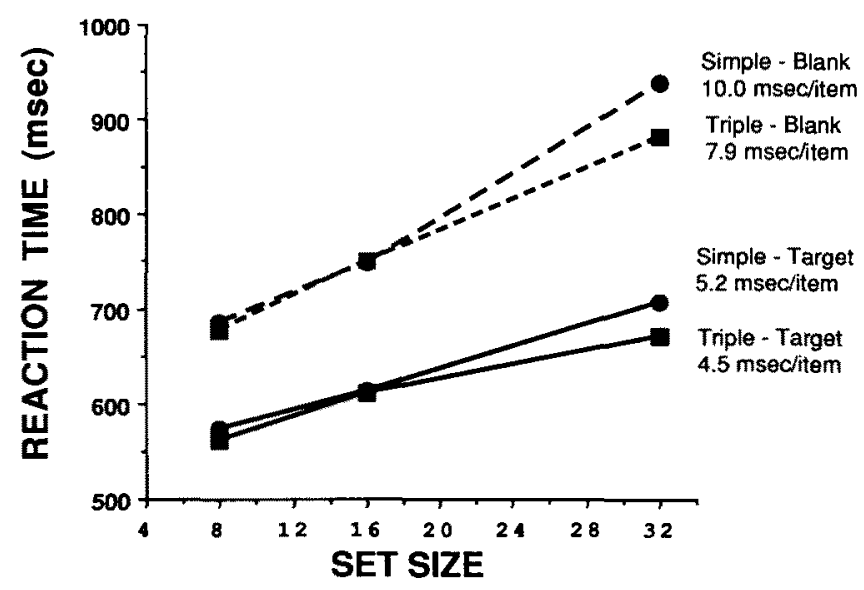

Figure 9. Comparison between results for simple conjunction and a triple conjunction in which each distractor shares two features with the target. (Slopes for triple conjunction are $4.5 \mathrm{~ms} / \mathrm{item}$ for target trials and $7.9 \mathrm{~ms} / \mathrm{item}$ for negative trials. Slopes for standard conjunction average $5.2 \mathrm{~ms} /$ item for target trials and $10.0 \mathrm{~ms} / \mathrm{item}$ for blank trials.)

directed to the most likely target. If the signal from the parallel processes is large relative to the noise, the target will be found quickly. If the signal is small, the spotlight will examine a number of distractor items before finding the target.

Triple conjunction searches where the target and distractors share only one feature produce shallower RT $\times$ Set Size functions than do standard conjunctions because there is more information available and presumably a larger signal sent from the parallel to the serial process. In other tasks, such as the $T$ versus $L$ task, the preattentive processes can provide no information. In the absence of a signal, the spotlight is guided only by the meaningless noise, and items are sampled at random until the target is found.

In a sense, it is no surprise to find that the preattentive processes guide the spotlight of attention (see Hoffman, 1978, 1979). The spotlight does not wander at random even in the standard feature integration model. It moves from stimulus item to item. If there were no guidance, the spotlight could spend time examining the blank regions in the display or it could wander free in the far periphery, well outside of the stimulus field. This does not happen because, at the very least, the preattentive processes inform later stages of the locations of stimuli. The guided search model suggests that the preattentive processes provide, in addition to mere positional information, information about the nature of individual items, at least to the extent of categorizing them as more or less worthy of attention.

Several questions remain to be answered. We do not know if the information from the preattentive processes is used to discard distractors, perhaps by inhibiting their spatial locations, or if, instead, it acts to label candidate targets, perhaps by enhancing their locations. Nor do we know if the preattentive processes supply their information only while the stimuli are visible or if that information can be used even if stimuli 


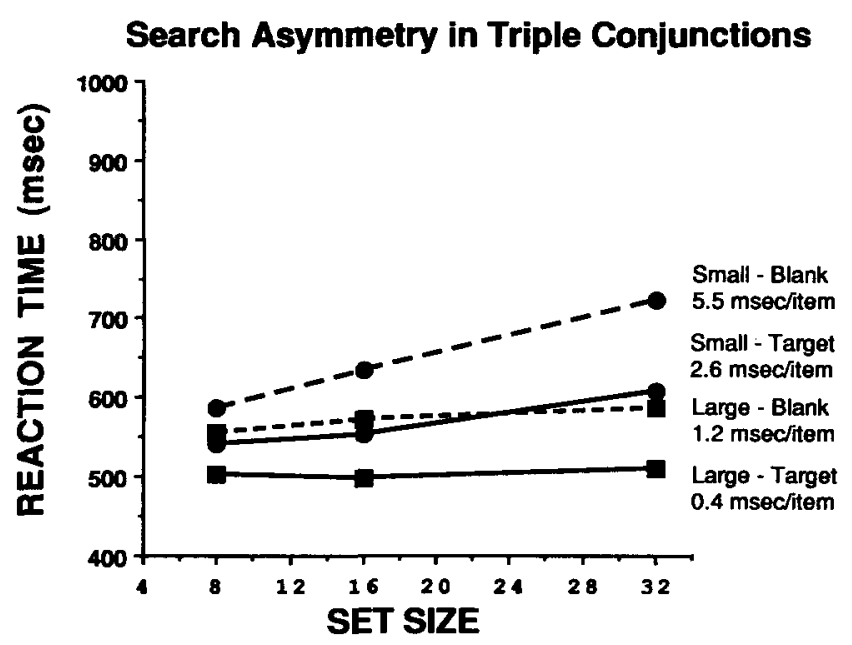

Figure 10. Comparison between two triple conjunction searches. (In both, each distractor shares one feature with the target. In the more efficient search, the target is large. In the other search, it is small. Slopes for triple conjunction search for large stimuli are 0.4 $\mathrm{ms} / \mathrm{item}$ for target trials and $1.2 \mathrm{~ms} / \mathrm{item}$ for blank trials. Slopes for triple conjunction search for small stimuli are $2.6 \mathrm{~ms} /$ item for target trials and $5.5 \mathrm{~ms} /$ item for blank trials.)

are briefly presented (as in Bergen \& Julesz, 1983; Julesz, 1984; Julesz \& Bergen, 1983). However, as discussed in the initial presentation of the model above, we assume that the parallel processes continue to send information to the serial process as long as the stimuli are visible. This ongoing updating allows a small signal to emerge from noise over time. A third unsolved problem pertains to search termination. We do not know how the subject decides when to give up a search and say "no" on a blank trial. In the case of a serial, selfterminating search, it is clear enough that the subject should search through each item and then quit, producing a 2:1 slope ratio. In parallel searches or in the hybrid parallel/serial searches that seem to characterize our conjunction experiments, it is unclear when the subject should end the search, and our data show wide variability in slope ratios.

A specific problem for the model is raised by the experiments of Nakayama and Silverman (1986). They used two targets and two distractors in their conjunction tasks, whereas we have only one type of target. For example, in a colorform conjunction task, such as that in Experiment 1 of this article, we might have a red $X$ as the target with green $X s$ and red $O$ s as distractors. In the Nakayama and Silverman experiments, the second target would be a green $O$. With red $X s$ and green $O$ s as targets, the parallel processes, it seems, would be unable to provide guidance to the serial process. Items could not be partitioned into distractors and candidate targets on the basis of color or form.

This problem rests on the assumption that subjects look for the red $X$ and green $O$ at the same time. If subjects perform two sequential searches, first for the red $X$, then for the green $O$, then the two-target case is reduced to two one-target searches of the sort described in this article. The parallel processes could first guide the search for the red $X$ and then, if necessary, guide the search for a green $O$. Preliminary data from our lab and from Treisman's (A. Treisman, personal communication) suggest that subjects are executing two sequential searches and do not search for both types of target at the same time.

\section{Conclusion}

We believe that the serial search for conjunctions can be guided by parallel processes that can divide the set of stimulus items into distractors and candidate targets. This allows naive subjects to search for conjunctions more efficiently than would be expected if it were necessary to do a full serial, selfterminating search. The efficiency of the search is a function of the quality of the guidance provided by the parallel processes. In the case of a triple conjunction (Experiment 9), the extra information from analysis of a third stimulus dimension allows for exceedingly efficient searches. A standard conjunction produces moderately efficient searches. In contrast, in a search for a $T$ among $L s$, there is no useful information in the parallel processes and the task reverts to a completely serial search. Our data can be accounted for within the feature integration model if and only if the parallel processes pass on useful information to guide the movements of the spotlight of attention.

\section{References}

Bergen, J. R., \& Julesz, B. (1983). Rapid discrimination of visual patterns. IEEE Transactions on Systems, Man, and Cybernetics, $S M C-13,857-863$.

Bundesen, C., \& Pedersen, L. F. (1983). Color segregation and visual search. Perception and Psychophysics, 33, 487-493.

Carter, R. C. (1982). Visual search with color. Journal of Experimental Psychology: Human Perception and Performance, 8, 127-136.

Cave, K. R., \& Wolfe, J. M. (in press). Modelling the role of parallel processing in visual search. Cognitive Psychology

Donders, F. C. (1969). On the speed of mental processes. Acta Psychologica, 30, (trans. by W. G. Koster), 412-431. (Original work published 1868)

Egeth, H. E., Virzi, R. A., \& Garbart, H. (1984). Searching for conjunctively defined targets. Journal of Experimental Psychology: Human Perception and Performance, 10, 32-39.

Farmer, E. W., \& Taylor, R. M. (1980). Visual search through color displays: Effects of target-background similarity and background uniformity. Perception \& Psychophysics, 27, 267-272.

Green, B. F., \& Anderson, L. K. (1956). Color coding in a visual search task. Journal of Experimental Psychology, 51, 19-24.

Hoffman, J. E. (1978). Search through a sequentially presented visual display. Perception \& Psychophysics, 23, 1-11.

Hoffman, J. E. (1979). A two-stage model of visual search. Perception \& Psychophysics, 25, 319-327.

Julesz, B. (1981). A theory of preattentive texture discrimination based on first order statistics of textons. Biological Cybernetics, 4I, $131-138$.

Julesz, B. (1984). A brief outline of the texton theory of human vision. Trends in Neuroscience, 7(2), 41-45.

Julesz, B., \& Bergen, J. R. (1983). Textons, the fundamental elements in preattentive vision and perceptions of textures. Bell Systems Technology, 62, 1619-1646.

McLeod, P., Driver, J., \& Crisp, J. (1988). Visual search for conjunctions of movement and form is parallel. Nature, 332, 154-155. 
Nakayama, K., \& Silverman, G. H. (1986). Serial and parallel processing of visual feature conjunctions. Nature, 320, 264-265.

Neisser, U. (1967). Cognitive psychology. New York: Appleton-Century-Crofts.

O'Connell, K. M., Treisman, A., \& Wolfe, J. M. (1988). [Density effects in visual search for conjunctions]. Unpublished raw data.

Pashler, H. (1987a). Detecting conjunctions of color and form: Reassessing the serial search hypothesis. Perception \& Psychophysics, 41, 191-201.

Pashler, H. (1987b). Target-distractor discriminability in visual search. Perception \& Psychophysics, 41, 285-292.

Quinian, P. T., \& Humphreys, G. W. (1987). Visual search for targats defined by combinations of color, shape, and size: An examination of the constraints on feature and conjunction searches. Perception \& Psychophysics, 41, 455-472.

Schneider, W., \& Eberts, R. (1980). Automatic processing and the unitization of $t$ wo features. Report No. 8008. Champagne-Urbana, IL: University of Illinois, Human Attention Research Laboratory.

Schneider, W., \& Shiffrin, R. M. (1977). Controlled and automatic human information processing: I. Detection, search, and attention. Psychological Review, 84, 1-66.

Shiffrin, R. M., \& Schneider, W. (1977). Controlled and automatic human information processing: Il. Perceptual learning, automatic attending, and a general theory. Psychological Review, 84, 127190.

Smith, S. L. (1962). Color coding and visual search. Journal of Experimental Psychology, 64, 434-440.
Steinman, S. B. (1987). Serial and parallel search in pattern vision. Perception, 16, 389-398.

Sternberg, S. (1969). High-speed scanning in human memory. Science, 153, 652-654.

Townsend, J. T. (1971). A note on the identification of parallel and serial processes. Perception \& Psychophysics, 10, 161-163.

Townsend, J. T. (1976). Serial and within-stage independent parallel model equivalence on the minimum completion time. Journal of Mathematical Psychology, 14, 219-239.

Treisman, A. (1986). Features and objects in visual processing. Scientific American, 255(11), 114B-125.

Treisman, A. (1988). Features and objects: The 14th Bartlett memorial lecture. Quarterly Journal of Experimental Psychology, 40A, 201-237.

Treisman, A., \& Gelade, G. (1980). A feature-integration theory of attention. Cognitive Psychology, 12, 97-136.

Treisman, A., \& Gormican, S. (1988). Feature analysis in early vision: Evidence from search asymmetries. Psychological Review, 95, 1548.

Treisman, A., and Paterson, R. (1984). Emergent features, attention, and object perception. Journal of Experimental Psychology: $\mathrm{Hu}$ man Perception and Performance, 10, 12-31.

Received April 4, 1988

Revision received July 29, 1988

Accepted July 29, 1988

\section{APA Convention "Call for Programs"}

The "Call for Programs" for the 1990 APA annual convention will be included in the October issue of the APA Monitor. The 1990 convention will be held in Boston, Massachusetts, from August 10 through August 14. Deadline for submission of program and presentation proposals is December 15,1989 . This earlier deadline is required because many university and college campuses will close for the holidays in mid-December and because the convention is in mid-August. Additional copies of the "Call" will be available from the APA Convention Office in October. 